\title{
Экспериментальные и теоретические исследования термоэлектрических свойств селенида меди
}

\author{
() Л.П. Булат ${ }^{1}$, В.Б. Освенский ${ }^{2}$, А.А. Иванов ${ }^{2,3, \uparrow}$, А.И. Сорокин ${ }^{2}$, Д.А. Пшенай-Северин ${ }^{4,5}$, \\ В.Т. Бублик ${ }^{6}$, Н.Ю. Табачкова ${ }^{6}$, В.П. Панченко ${ }^{2,6}$, М.Г. Лаврентьев ${ }^{2}$ \\ ${ }^{1}$ Университет ИТМО, \\ 191002 Санкт-Петербург, Россия

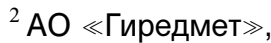 \\ 119017 Москва, Россия \\ ${ }^{3}$ Московский технологический университет, \\ 119454 Москва, Россия \\ ${ }^{4}$ Физико-технический институт им. А.Ф. Иоффре Российской академии наук, \\ 194021 Санкт-Петербург, Россия \\ ${ }^{5}$ Санкт-Петербургский политехнический университет Петра Великого, \\ 195251 Санкт- Петербург, Россия \\ ${ }^{6}$ Национальный исследовательский технологический университет «МИСиС», \\ 119991 Москва, Россия \\ I E-mail: girlab22@mail.ru
}

(Получена 27 декабря 2016 г. Принята к печати 12 января 2017 г.)

\begin{abstract}
Проведено экспериментальное и теоретическое исследование температурных зависимостей теплоемкости, теплопроводности, коэффициента теплового расширения и кинетических коэффициентов (электропроводности, коэффициента термоэдс) селенида меди в интервале температур 300-873 K. Соответствие результатов расчета и эксперимента наблюдается до температуры $\sim 773 \mathrm{~K}$. Величина максимальной термоэлектрической эффективности в наноструктурированном селениде меди находится на уровне $Z T \sim 1.8$. Продемонстрирована корреляционная зависимость $Z T$ от величины теплопроводности во всем исследованном температурном интервале.
\end{abstract}

DOI: $10.21883 /$ FTP.2017.07.44635.21

\section{1. Введение}

Селенид меди является перспективным термоэлектрическим материалом для термоэлектрических генераторов (ТЭГ) и обладает исключительно высокой термоэлектрической эффективностью. В ряде работ $[1,2]$ сообщалось о получении $\mathrm{Cu}_{2} \mathrm{Se}$ с добротностью $Z T$ от 1.2 до 1.6 при температурах от 800 до $1000 \mathrm{~K}$. Считается, что такие высокие значения связаны прежде всего с аномально низкой теплопроводностью селенида меди при высоких температурах, что объясняется высокой подвижностью ионов меди. В настоящей работе проведено теоретическое и экспериментальное исследование теплоемкости, теплопроводности, коэффициента теплового расширения и кинетических коэффициентов (электропроводности, коэффициента термоэдс) селенида меди.

\section{2. Методика эксперимента}

Материалы для исследования получали по технологической схеме, успешно использованной нами для получения наноструктурных материалов на основе $\mathrm{Bi}_{2} \mathrm{Te}_{3}[3,4]$. Нанопорошок селенида меди был получен методом механохимического синтеза (MXC) в высокоэнергетической шаровой мельнице при 300 об/мин и времени синтеза 2-10ч. В качестве исходных материалов использовали порошок $\mathrm{Cu}(99.9 \%)$ и гранулы $\mathrm{Se}(99.999 \%)$. Синтезированный материал компактировали методом искрового плазменного спекания (ИПС) при температуpe $550^{\circ} \mathrm{C}$, давлении 50 МПа и времени выдержки 10 мин.

\section{3. Результаты и их обсуждение}

Одной из возможных причин повышения ZT считают снижение теплоемкости с ростом температуры [4]. На основе теоретического расчета фононного спектра в кубической $\beta$-фазе $\mathrm{Cu}_{2} \mathrm{Se}$ была предложена теория теплоемкости этого материала. В данном расчете учтено снижение теплоемкости, которое может иметь место из-за высокой подвижности ионов меди. В $\mathrm{Cu}_{2} \mathrm{Se}$ подрешетка селена с ростом температуры сохраняет свойства, присущие твердым телам, а подрешетка более подвижных ионов меди оказывается разупорядоченной и приближается по своим свойствам к жидкости. Поскольку теплоемкость жидкости ниже теплоемкости твердых тел, в селениде меди можно предполагать снижение теплоемкости с температурой. Для объяснения снижения теплоемкости была использована теория Траченко [5], основанная в свою очередь на теории жидкости Френкеля [6]. В рамках этого подхода для описания свойств жидкости в качестве исходной точки 
используется твердое тело. Увеличение вероятности перескоков атомов при повышении температуры приводит к тому, что в жидкости исчезает часть поперечных мод, что становится причиной снижения теплоемкости. Поскольку считается, что в $\mathrm{Cu}_{2} \mathrm{Se}$ только подрешетка атомов меди переходит в состояние, сходное с жидким, были выделены не только продольная и поперечная составляющие колебаний, но и доли вкладов атомов меди и селена в теплоемкость.

Результаты измерения теплоемкости $C_{p}$ в интервале температур до $973 \mathrm{~K}$ показывают, что величина $C_{p}$ практически не изменяется (снижение находится на уровне $5 \%$ ), что согласуется с расчетной температурной зависимостью теплоемкости с учетом частоты перескоков, определенной по коэффициенту самодиффузии меди. Более подробно вопрос теплоемкости селенида меди обсуждается в нашей работе [7].

С целью выяснения механизма снижения теплопроводности в селениде меди были проведены теоретические расчеты температурной зависимости теплопроводности с использованием метода равновесной молекулярной динамики и классического межатомного потенциала.

Согласно расчету, величина решеточной теплопроводности составляет $\sim 0.6 \mathrm{BT} / \mathrm{M} \cdot \mathrm{K}$ и слабо изменяется с температурой. Результаты моделирования подтвердили высокую подвижность ионов меди. Это позволяет заключить, что исключительно низкое значение решеточной теплопроводности в $\mathrm{Cu}_{2} \mathrm{Se}$ обусловлено сильным рассеянием фононов на подвижных атомах $\mathrm{Cu}$.

Было проведено также экспериментальное исследование температурной зависимости полной $\kappa$ и решеточной $\kappa_{\text {lat }}$ теплопроводности. Решеточную теплопроводность $\kappa_{\text {lat }}$ рассчитывали по следующим формулам:

$$
\begin{gathered}
\kappa_{\text {lat }}=\kappa-\kappa_{e}, \\
\kappa_{e}=L \sigma T,
\end{gathered}
$$

где $\kappa_{e}-$ электронная теплопроводность, $L=1.5 \cdot 10^{-8} \mathrm{BT} \cdot \mathrm{Oм} / \mathrm{K}^{2}-$ число Лоренца, $\sigma-$ электропроводность, $T-$ абсолютная температура.

На рис. 1 представлена температурная зависимость полной $\kappa$ и решеточной $\kappa_{\text {lat }}$ теплопроводности для объемного наноструктурированного образца $\mathrm{Cu}_{2} \mathrm{Se}$, полученного методом ИПС. Там же, для сравнения, представлены литературные данные из [1]. Экспериментально полученная полная теплопроводность уменьшается с повышением температуры, особенно сильно при $T>800 \mathrm{~K}$, и при $T=900 \mathrm{~K}$ составляет $\sim 0.62 \mathrm{Bт} / \mathrm{M} \cdot \mathrm{K}$. Решеточная теплопроводность существенно ниже $\kappa$, особенно при температуре до $700 \mathrm{~K}$. При $T=900 \mathrm{~K} \kappa_{\text {lat }}$ в образцах, полученных методом ИПС, составляет $\sim 0.25 \mathrm{BT} / \mathrm{M} \cdot \mathrm{K}$. В интервале температур до $\sim 750 \mathrm{~K} \kappa_{\text {lat }}$ изменяется слабо.

При расчете температурной зависимости коэффициента термоэдс $(\alpha)$ и электропроводности $(\sigma)$ были учтены энергетическая и температурная зависимости времени релаксации, которая соответствует наиболее сильному и

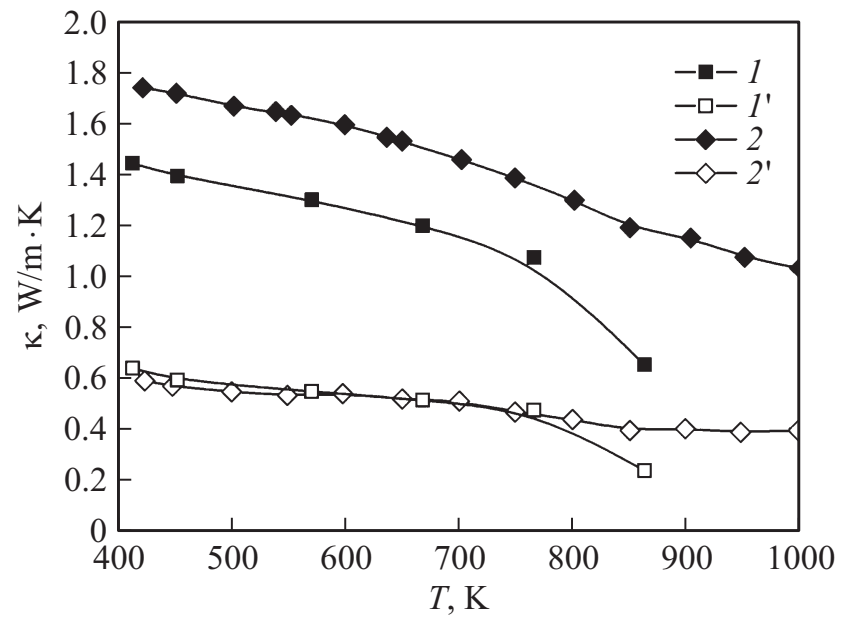

Рис. 1. Температурная зависимость теплопроводности $\kappa$. $1,1^{\prime}$ - полная теплопроводность и ее решеточная составляющая наноструктурированных образцов, полученных в данной работе; 2, 2' - полная теплопроводность и ее решеточная составляющая из [1].

часто встречающемуся механизму рассеяния на акустических фононах.

На рис. 2 приведены расчетные зависимости $\sigma(a)$ и $\alpha(b)$ в $\beta$ - $\mathrm{Cu}_{2} \mathrm{Se} p$-типа. Там же представлены, полученные нами, экспериментальные данные. В целом удовлетворительное соответствие расчета и эксперимента имеется в интервале 450-773 К. При более высоких температурах экспериментальные значения термоэдс резко возрастают, а электропроводность падает. Если предположить, что концентрация дырок остается постоянной, то это соответствует увеличению эффективной массы носителей с температурой.

Возможные причины возрастания эффективной массы заключаются в сложной зонной структуре, непараболичности спектра, а также в тепловом расширении кристалла. Использованный в данной работе расчет учитывает непараболичность спектра и наличие более глубоких валентных зон. Для того чтобы проиллюстрировать влияние теплового расширения, был рассчитан электронный спектр в $\mathrm{Cu}_{2} \mathrm{Se}$ при экспериментальных значениях постоянной решетки в диапазоне $700-1000 \mathrm{~K}$ по данным работы [8]. Расчет показал, что изменение энергетического спектра с учетом теплового расширения кристалла приводит к увеличению эффективной массы носителей и коэффициента термоэдс. Однако его величина недостаточна для объяснения экспериментальных зависимостей. Дополнительный вклад, возможно, связан с изменением спектра за счет усиления разупорядочения атомов в решетке при более высокой температуре.

Изучение поведения точечных дефектов при нагреве селенида меди можно осуществить путем измерения теплового расширения и температурной зависимости периода решетки. Результаты исследования теплового расширения термоэлектрического материала $\mathrm{Cu}_{2} \mathrm{Se}$ при- 

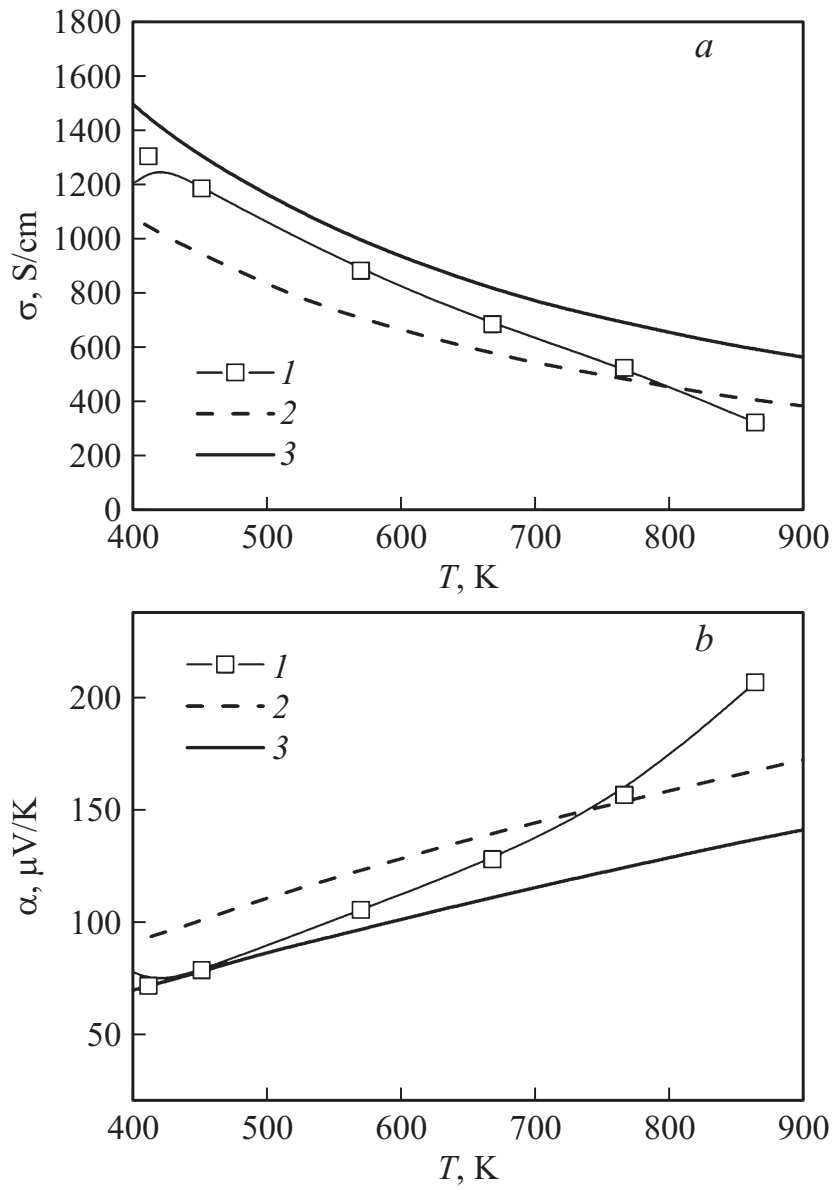

Рис. 2. $a-$ экспериментальная (1) и расчетные $(2,3)$ температурные зависимости электропроводности $\sigma$ для концентраций дырок $4 \cdot 10^{20} \mathrm{~cm}^{-3}(2)$ и $6 \cdot 10^{20} \mathrm{~cm}^{-3}(3)$ в $\mathrm{Cu}_{2} \mathrm{Se}$ $p$-типа. $b$ - экспериментальная $(1)$ и расчетные $(2,3)$ температурные зависимости термоэдс $\alpha$ для концентраций дырок $4 \cdot 10^{20} \mathrm{~cm}^{-3}(2)$ и $6 \cdot 10^{20} \mathrm{~cm}^{-3}(3)$ в $\mathrm{Cu}_{2} \mathrm{Se} p$-типа.

ведены на рис. 3. На нем верхняя кривая - коэффициент термического расширения (КТР), нижняя относительное удлинение. Из рисунка видно, что после завершения фазового перехода первого рода, начиная приблизительно с $473 \mathrm{~K}$, тепловое расширение характеризует высокотемпературную кубическую фазу. Характерно, что при температуре до 673 К КТР растет, как это обычно наблюдается для твердых тел при температуре выше температуры Дебая. Однако при температуре выше 673 К КТР не только не растет, но и несколько уменьшается вплоть до температуры $\sim 773 \mathrm{~K}$. Такое изменение КТР может быть связано с изменением состояния точечных дефектов, в частности с образованием вакансий селена в результате сублимации $\mathrm{Cu}_{2} \mathrm{Se}$ при нагреве. Измерение интенсивности максимумов на дифрактограммах, снятых при разных температурах, позволило оценить факторы Дебая-Уоллера и определить среднеквадратичные смещения отдельно атомов $\mathrm{Cu}$ и $\mathrm{Se}$. Значение среднеквадратичного смещения $\mathrm{Cu}$ оказалось примерно в 4 раза больше. Это свидетельствует о том, что коэффициент диффузии у меди больше, а энтальпия образования вакансий меньше, чем у селена.

По положению брэгговского пика на дифрактограмме при разных температурах от 473 до $673 \mathrm{~K}$ найдена зависимость параметра решетки от температуры. При изменении температуры выше $673 \mathrm{~K}$ рост параметра решетки несколько замедляется.

Наличие перегибов при температуре $\sim 773 \mathrm{~K}$ на температурных зависимостях $C_{p}$, КТР, $\kappa, \sigma, \alpha$ в [4] объясняется тем, что она является нижней температурной границей разупорядочения ионов $\mathrm{Cu}^{+}$(по аналогии со стеклом) без фазового перехода первого рода. При этом изменяется ионное состояние $\mathrm{Cu}$ (от $\mathrm{Cu}-\mathrm{Se}$ $\left.\kappa(\mathrm{Cu}-\mathrm{Cu})^{\circ}\right)$.

На рис. 4 приведена, полученная в данной работе, экспериментальная температурная зависимость ZT для образцов $\mathrm{Cu}_{2} \mathrm{Se}$, полученных методом ИПС. При температуре $870 \mathrm{~K}$ величина $Z T$ достигает 1.8 , что значительно выше термоэлектрической эффективности материала, полученного другими методами $[1,2,9]$. Как

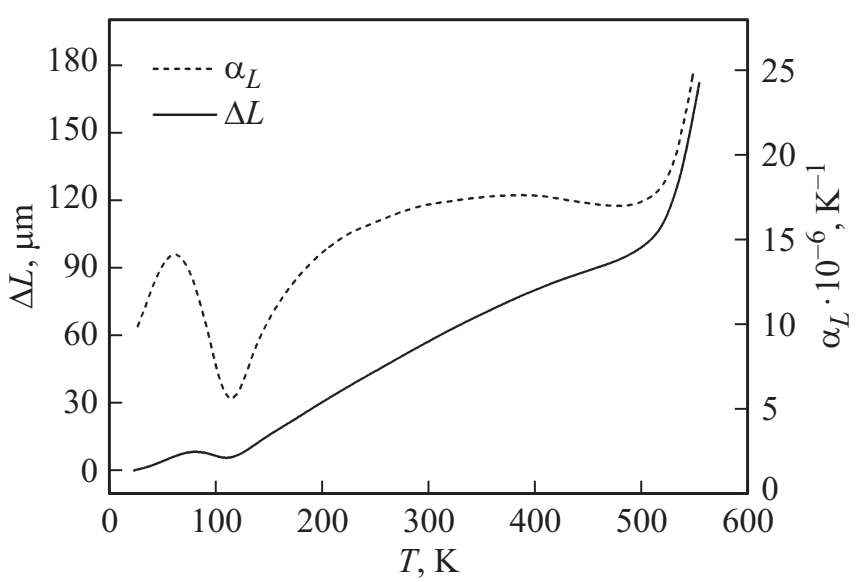

Рис. 3. Температурная зависимость коэффициента теплового расширения $\alpha_{L}$ и относительного удлинения $\Delta L$ селенида меди.

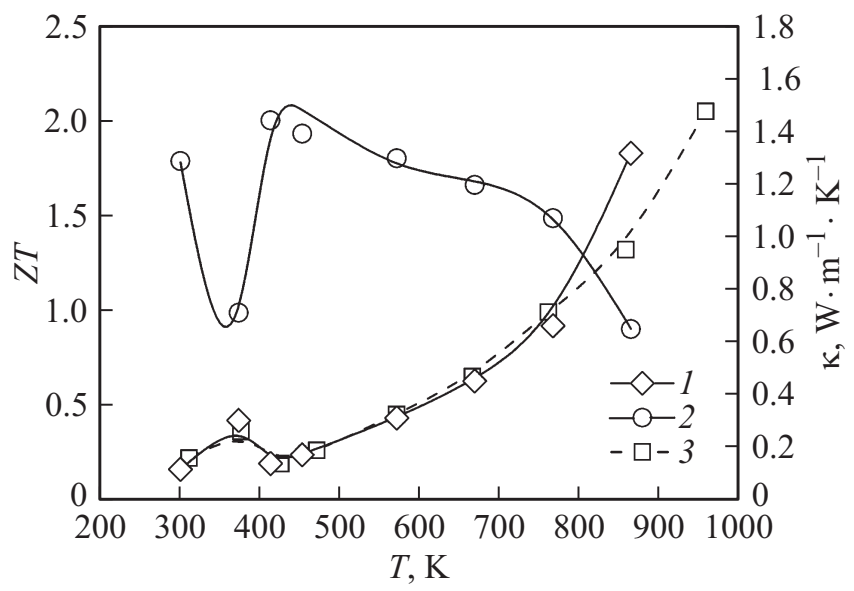

Рис. 4. Полученная в данной работе температурная зависимость $Z T$ (1) и $\kappa(2) ; Z T$ по данным [9] (3). 
указывалось выше, отличительной особенностью материала, полученного методом ИПС, является то, что он имеет нанокристаллическую структуру. На рис. 4 также представлена температурная зависимость $Z T$ наноструктурированного материала, полученного по аналогичной технологической схеме [10], где $Z T$ при $950 \mathrm{~K}$ достигает 2.0. Это свидетельствует о том, что наноструктурированный $\mathrm{Cu}_{2} \mathrm{Se}$ обладает более высокой термоэлектрической эффективностью. Приведенные на рис. 4 данные подтверждают наличие корреляционной зависимости $Z T$ от величины теплопроводности. Это говорит о том, что основной причиной высоких значений $Z T$ является низкая теплопроводность решетки, связанная с высокой подвижностью ионов меди, что согласуется с результатами расчета.

\section{4. Заключение}

Проведены экспериментальные и теоретические исследования температурных зависимостей теплоемкости, теплопроводности, а также кинетических коэффициентов (электропроводности, коэффициента термоэдс) и их вклада в термоэлектрическую эффективность селенида меди. Удовлетворительное соответствие экспериментальных и расчетных данных наблюдается при температуре до $\sim 773 \mathrm{~K}$, после чего на экспериментальных кривых температурной зависимости наблюдается перегиб. Немонотонное изменение коэффициента термического расширения при температуре от 400 до $800 \mathrm{~K}$ может быть связано с изменением состояния точечных дефектов. Величина ZT наноструктурированных образцов $\mathrm{Cu}_{2} \mathrm{Se}$, полученных методом ИПС, при температуре от 850 до $950 \mathrm{~K}$ может достигать 1.8 и даже 2.0 (по данным [10]). Наблюдается наличие корреляционной зависимости ZT от величины теплопроводности, что согласуется с результатами теоретического расчета.

Работа выполнена при поддержке Министерства образования и науки РФ (субсидия № RFMEFI57914X003914.579.21.0039).

\section{Список литературы}

[1] H. Liu, X. Shi, F. Xu, L. Zhang., W. Zhang, L. Chen, Q. Li, C. Uher, T. Day, G. J. Snyder. Nature Materials, 11, 422 (2012).

[2] B. Yu, W. Liu, S. Chen, H. Wang, H. Wang, G. Chen, Z. Ren. Nano Energy, 1 (3), 472 (2012).

[3] L.P. Bulat, D.A. Pshenay-Severin, V.V. Karatayev, V.B. Osvenskii, M. Lavrentev, A. Sorokin, V.D. Blank, G.I. Pivovarov, V.T. Bublik, N.Yu. Tabachkova. In: A.A. Hashim (ed). The Delivery of Nanoparticles. In Tech: 454 (2012).

[4] L.P. Bulat, V.T. Bublik, I.A. Drabkin, V.V. Karatayev, V.B. Osvenskii, Yu.N. Parkhomenko, G.I. Pivovarov, D.A. PshenaiSeverin, N.Yu. Tabachkova. J. Electron. Mater., 39(9), 1650 (2012).

[5] K. Trachenko. Phys. Rev. B, 78, 104201 (2008).
[6] J. Frenkel. Kinetic Theory of Liquids, ed. by R.H. Fowler, P. Kapitza, N.F. Mott (Oxford, Oxford University Press, 1947).

[7] L.P. Bulat, D.A. Pshenay-Severin, A.A. Ivanov, V.B. Osvenskii, Yu.N. Parkhomenko. J. Electron. Mater. (First Online: 07 October 2016, DOI: 10.1007/s11664-016-4967-6).

[8] H. Kim, S. Ballikaya, H. Chi, J.-P. Ahn, K. Ahn, C. Uher, M. Kaviany. Acta Mater., 86, 247 (2015).

[9] T.W. Day, K.A. Borup, T. Zhang, F. Drymiotis, D.R. Brown, X. Shi, L. Chen, B.B. Iversen, G.J. Snyder. Mater. Renew. Sustain Energy, 3 (2), 26 (2014).

[10] B. Gahtori, S. Bathula, K. Tyagi, A.K. Srivastava, A. Dhar, R.C. Budhani. Patent USA № WO 2015037014 A1 (2015).

Редактор Г.А. Оганесян

\section{Experimental and theoretical study of the thermoelectric properties of copper selenide}

L.P. Bulat ${ }^{1}$, V.B. Osvenskii' ${ }^{2}$, A.A. Ivanov ${ }^{2,3}$, A.I. Sorokin ${ }^{2}$, D.A. Pshenay-Severin ${ }^{4,5}$, V.T. Bublik ${ }^{6}$, N.Yu. Tabachkova ${ }^{6}$, V.P. Panchenko ${ }^{2,6}$, M.G. Lavrentev ${ }^{2}$

${ }^{1}$ ITMO University, 191002 St. Petersburg, Russia

2 "Giredmet“ JSC,

119017 Moscow, Russia

${ }^{3}$ Moscow Technological University (MIREA), 119454 Moscow, Russia

${ }^{4}$ Ioffe Institute, Russian Academy of Science, 194021 St. Petersburg, Russia

${ }_{5}^{5}$ Peter the Great St. Petersburg Polytechnical University, 195251 St. Petersburg, Russia

${ }^{6}$ National University of Science and Technology „MISiS“,

119991 Moscow, Russia

\begin{abstract}
Experimental and theoretical study of temperature dependences of heat capacity, thermal conductivity, coefficient of thermal expansion and transport coefficients (electrical conductivity and thermopower) of copper selenide were carried out in temperature range $300-873 \mathrm{~K}$. The results of calculations correlate with experimental data up to $\sim 773 \mathrm{~K}$. The maximum value of thermoelectric efficiency of nanostructured copper selenide is $Z T \sim 1.8$. The correlation of $Z T$ and thermal conductivity were demonstrated within investigated range of temperatures.
\end{abstract}

